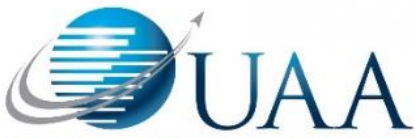

Collegiate Aviation Review

International

UNIVERSITY AVIATION

ASSOCIATION

\title{
Utilizing UAS to Support Wildlife Hazard Management Efforts by Airport Operators
}

\author{
Flavio A. C. Mendonca \\ Embry-Riddle Aeronautical University
}

\author{
Ryan Wallace \\ Embry-Riddle Aeronautical University
}

The FAA requires airports operating under the Code of Federal Regulations Part 139 to conduct a wildlife hazard assessment (WHA) when some wildlife-strike events have occurred at or near the airport. The WHA should be conducted by a Qualified Airport Wildlife Biologist (QAWB) and must contain several elements, including the identification of the wildlife species observed and their numbers; local movements; daily and seasonal occurrences; and the identification and location of features on and near the airport that could attract wildlife. Habitats and landuse practices at and around the airport are key factors affecting wildlife species and the size of their populations in the airport environment. The purpose of this ongoing study is to investigate how UAS technologies could be safely and effectively applied to identify hazardous wildlife species to aviation operations as well as potential wildlife hazard attractants within the airport jurisdiction. Researchers have used a DJI Mavic 2 Enterprise Dual drone with visual and thermal cameras to collect data. Data have been collected in a private airport in a "Class G" airspace. We have applied different risk mitigation strategies to mitigate risks associated with drone operations in an airport environment, including a visual observer during data collection, and an ADS-B flight box to obtain information of manned aircraft at and around the airport. Multiple flights were conducted in different days of the week as well as different times of the day. Noteworthy to mention we have had the technical support of QAWB throughout this study. Preliminary findings suggest that UAS can facilitate the observations made by a QAWB during a WHA, including the identification and assessment of potential wildlife attractants (e.g., wetlands), and the identification of wildlife species (e.g., White ibis). Additionally, initial findings indicate that UAS facilitates data collection in areas that are difficult to access by ground-based means (e.g., wetlands). Another key finding of this study was that our team could observe, and with the assistance of the QAWB identify different wildlife species and habitats simultaneously during each UAS flight. In different words, from a single image (video and/or picture) a QAWB could obtain valuable information about different wildlife species and related habitats. Lastly, results suggest that the versatility and speed of UAS (including their high-quality cameras and sensors) ensure that data can be collected more thoroughly and faster over large areas during a WHA.

\section{Recommended Citation:}

Mendonca, F. A. C., \& Wallace, R. (2021). Utilizing UAS to support wildlife hazard management efforts by airport operators. Collegiate Aviation Review International, 39(2), 238-248. Retrieved from

http://ojs.library.okstate.edu/osu/index.php/CARI/article/view/8385/7686 


\section{Introduction}

Aircraft accidents resulting from wildlife strikes have become an increasing economic and safety concern for the U.S. aviation industry. Wildlife strikes have killed more than 295 people and destroyed over 271 worldwide since 1988. According to Dolbeer et al. (2021), there were 238,654 wildlife strikes in the U.S. from 1990 through 2018 . Almost $97 \%$ of these strikes involved birds. During this period, 16 and 251 wildlife strikes resulted in 36 fatalities and 327 people injured, respectively. Seventy-four aircraft were damaged beyond repair due to strikes. Annually, conservative estimates suggest that wildlife strikes cost on average \$200 million in monetary losses and 110,000 hours of aircraft downtime. Nonetheless, previous studies (DeVault et al., 2018; Dolbeer et al., 2021) have suggested that frequently wildlife strike reports do not provide accurate information regarding the direct and other monetary costs estimates. Yet, many strikes go unreported. Thus, it can be assumed the costs of wildlife strikes could be significantly higher.

Certificated airports are required to complete a wildlife hazard assessment when certain safety events (e.g., multiple strikes involving an air carrier) have occurred at or near an airport. This process should be conducted by a qualified airport wildlife biologist (QAWB). The Title 14 Code of Federal Regulations (CFR) Part 139.337 prescribes the required elements of a WHA, which include the identification of wildlife species to aviation operations as well as the location of habitats and other features that could attract hazardous wildlife species to the airport air operations area (AOA) (Cleary \& Dolbeer, 2005). A WHA provides the foundation for an effective wildlife hazard management plan (WHMP). The FAA has provided guidance on the protocols for conducting a WHA and WHMP (FAA, 2018), on land uses and other habitats with the potential to attract hazardous wildlife (FAA, 2020) to the airport environment, and on the most hazardous wildlife species to aviation operations.

Safety programs and other strategies by aviation stakeholders have certainly improved aviation safety. Notwithstanding, information obtained from the analyses of wildlife strike data has indicated that mitigating the risk of accidents due to wildlife strikes will present unique challenges over the next decades. For example, the number of strikes has increased from 9,840 in 2011 to 17,178 in 2019 (a 75\% increase). Similarly, the number of damaging strikes increase by $41 \%$ over the same period. The rate of strikes and of damaging strikes per million aircraft movements increased by $42 \%$ and by $7 \%$ from 2011 through 2019 . For GA and commercial aircraft, $87 \%$ and $82 \%$ of the total strikes, respectively, occurred at the airport environment (at or below 1,500 above ground level [AGL]) (Dolbeer et al., 2021).

The effective management of wildlife hazards is a defense in depth, and includes actions by pilots, aircraft and airport certification standards, and safety programs by airport operators (Mendonca et al., 2018). A multifaceted approach for mitigating strikes is vital, and that should include certification standards for aircraft airframe and engines, outreach, and education 
(Eschenfelder \& DeFusco, 2010). Additionally, it should include the use of new technologies and/or innovative approaches to current technologies. Hamilton et al. (2020a, 2020b) and Prather (2019) have also suggested the investigation of unmanned aircraft systems (UAS) for identifying hazardous wildlife species to aviation operations.

UAS has been experiencing healthy growth in the U.S. over the past years. In fact, UAS has have been used for multiple purposes, ranging from recreation to data collection during research projects. According to Prather (2019), new applications of UAS are frequently being established around the world. UAS application at the airport environment could include construction, security, and emergency management; and wildlife hazard mitigation. Nonetheless, the safe incorporation of UAS and related technologies into an airport environment, still in early stages, presents major safety, economic, regulatory, and infrastructure challenges. Nonetheless, it also presents opportunities and benefits to include cost efficiencies, increased mission effectiveness, and career opportunities. Hamilton et al. (2020a) have indicated that research is needed to evaluate the effectiveness of using UAS in the airport environment. Moreover, empirical data and information can help develop metrics to "determine how the UAS will benefit the airport" (Prather, 2019, p. 40).

To address a gap in previous research, the current study is an attempt to investigate how UAS can be used to support the airport operator's efforts to mitigate the risk of aircraft accidents resulting from wildlife strikes. More specifically, the goals of this ongoing exploratory study are to:

1. Identify the most effective UAS and sensors for data collection during a WHA;

2. Explore best practices for UAS application to identify hazardous wildlife species and related habitats at the airport environment; and

3. Develop operational procedures and workflows that support the effective application of UAS at the airport environment during a WHA.

\section{Concept of Operations}

Hamilton et al. (2020a) defined concept of operations (CONOPS) for UAS as "a description of the nature of UAS operations and the resulting impacts on relevant stakeholders and the environment" (p. 3). The ConOps include the UAS flight pattern, regulatory requirements, and safety risk management processes and procedures. Our team utilized Hamilton et al. (2020a, 2020b), Neubauer et al. (2015), and Prather (2019) while developing (and updating) the ConOps for the current study. It is important to mention that a QAWB has provided expertise and guidance throughout this study.

During the first phase of this study (spring 2021) we utilized a DJI Mavic 2 Enterprise drone with visual and thermal cameras. The integrated thermal-visual cameras have multiple functionalities which allowed our team to switch between thermal, visual, or even split-view feeds. The ground position system (GPS) location, time, and date of images (photos and videos) were recorded on GPS timestamping (see Figure 1). 


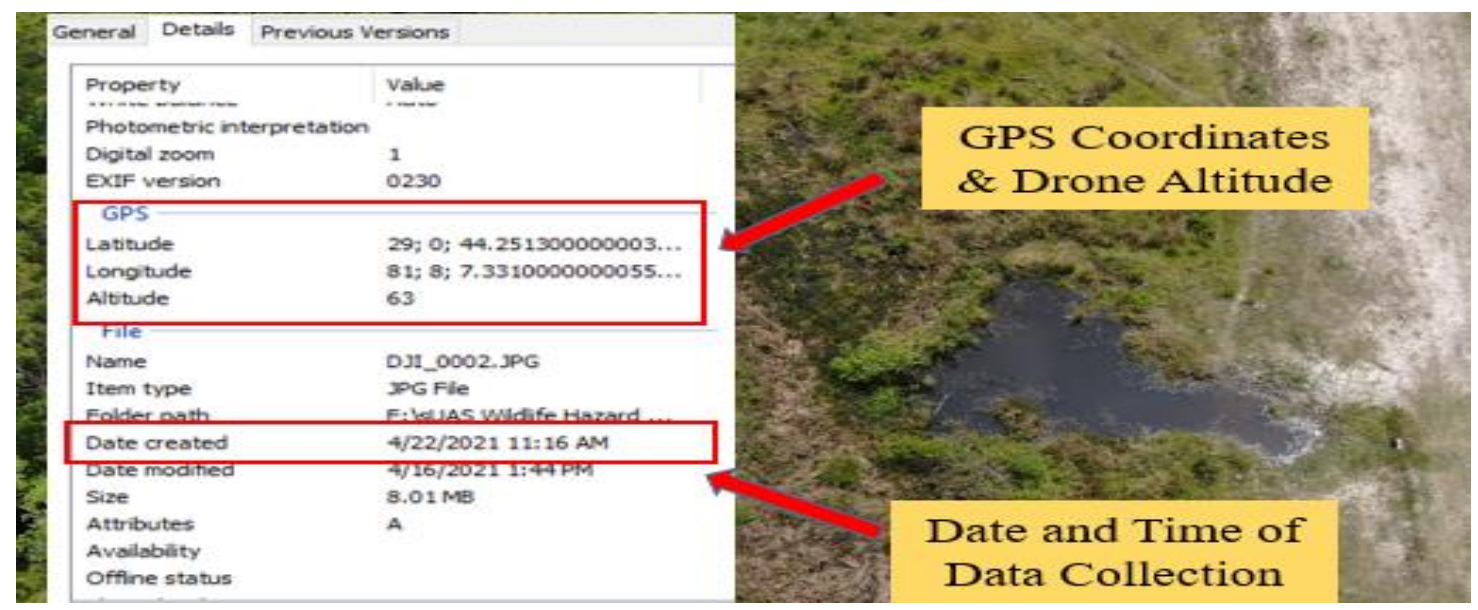

Figure 1. GPS Coordinates, Date and Time, And Altitude of Flight for Each Image Were Recorded

Data were collected on April 16/29/30 and on May 14 at Coe Field, a private use general aviation airport located in a Class G airspace. Coe Field is located approximately 11 nautical miles southwest of Daytona Beach International Airport (KDAB) at the latitude of $29^{\circ}$ 00' 37" N and longitude of $81^{\circ} 07^{\prime}$ '56" W. According to Cleary and Dolbeer (2005), habitats and land-use practices are important elements determining the wildlife species and the size of their populations being attracted to the airport jurisdiction. Yet, land use (e.g., agricultural activity), as well as habitats (e.g., wetlands), will influence the behavior of the wildlife species attracted to the airport environment. Coe Field, which is located in a rural area, is surrounded by trees, farmland, and bodies of water that attract various wildlife species. It is noteworthy to mention that the airport owner raises cattle in the airport premises, which also favors the presence of flocking birds (Cornell Lab of Ornithology, 2021a, 2021b).

Researchers utilized a trailer with different pieces of equipment that included an automatic detection surveillance broadcast (ADS-B) flight box that transmitted a Wi-Fi network that is connectable by a tablet of mobile; two television (TV) sets; and a monitoring station. Once the Wi-Fi network was connected by an electronic device (usually a cell phone), researchers used ForeFlight (ForeFlight, 2021) to identify and monitor manned aircraft at and around the data collection area. The electronic device was connected to a TV (inside the trailer) via a high-definition multimedia interface (HDMI) cable. This process helped our team mitigate the risk associated with UAS operations in an airport environment since we could identify the location, altitude, heading, and speed of any aircraft displayed on the live traffic feed. We determined that if manned aircraft were observed approaching Coe Field at or below 1,000 feet (ft) above ground level (AGL), the drone would be manually flown at a safer (lower) altitude and brought back to the pilot's position. Further risk mitigation strategies associated with manned aircraft operations our team implemented included:

1. All UAS flights were conducted at or below $200 \mathrm{ft}$ AGL;

2. No UAS flights were conducted in the departure, approach, and circling airspaces of the airport;

3. All UAS flights were only conducted during visual meteorological conditions (VMC) and with a visibility of at least 5,000 NM; and 
4. A visual observer whose roles included ensuring the drone was always within line of sight as well as warning the drone's pilot of the presence of manned aircraft in the data collection area;

In addition, a flight risk assessment tool was exercised before each flight so that our team could identify hazards, and develop and implement safety measures to mitigate the associated risks (FAA, 2016). Some hazards may not be identified, nor the associated risks are effectively mitigated. Thus, safety measures and aircraft performance margins were built into the ConOps. Most importantly, the ConOps was frequently revised and updated accordingly in order to ensure aviation safety and efficiency, as suggested by Hamilton (2020a, 2020b).

The researchers developed a Wildlife Survey Observation Sheet (WSOS) with the support of the QAWB to record data during data collection. This observation form allowed our team to follow a similar protocol utilized by a QAWB during a WHA. Data were collected in an area of approximately 300x300 meters north of Coe Field. Multiple flights were conducted during four days of data collection so that researchers could capture daily and other factors affecting the presence of and/or the behavior of the observed wildlife species as suggested by the FAA (2018). The "DJI's Go 4 Software", used through the smart drone controller, allowed the drone's pilot to create flight plans as well as to store telemetry data from each flight. The smart controller was hooked up via an HDMI cable to the other TV set inside the trailer, where the outside elements would not affect what was being observed. At least one member of our team stayed in the monitoring station inside the trailer observing the two TV sets and writing down observed wildlife activities, the presence of wildlife attractants, and possible interactions between those elements. In addition, this person monitored possible manned aircraft activities at or close to the data collection area. This process not only enhanced our data collection process but also increased the safety of the UAS operations.

The first and third flights were conducted autonomously in a basic grid pattern at an average height of $150 \mathrm{ft}$ AGL. The second and fourth flights were carried out manually based upon the drone's pilot's and team members' (especially the team's member in the monitoring station) observations during the autonomous flights. The manual flights allowed our team to collect images from different altitudes, angles, cameras' parameters. They also allowed our team to identify and especially explore elements (e.g., wildlife interactions with habitats) that are vital during a WHA (FAA, 2018). This multiplicity of flights using different patterns "allowed our team to collect several images that overlapped, giving researchers (and the QAWB) a better and more accurate picture of wildlife hazards and of possible wildlife hazard attractants, and the interactions between those elements" (Cabrera et al., 2021, p.14). Moreover, it allowed the researchers to observe and collect multiple images of a specific area; wildlife species (to include their numbers and locations, local movements, and behaviors); and/or wildlife hazard attractants from multiple viewpoints.

\section{Key Findings}

There were 5,020 and 1,195 strikes and damaging strikes involving mammals in the U.S., respectively, from 1990 through 2020. Twenty-nine aircraft were destroyed after colliding with mammals. Three of them involved cattle. Thirty-one and one people were injured and killed, 
respectively, due to strikes with mammals. The direct and other monetary economic losses resulting from those strikes were estimated to be almost $\$ 70$ million (Dolbeer et al., 2021). As previously noted, the airport's owner raises cattle at Coe Field. Analyses of wildlife strike data have indicated that Cattle have not been a safety hazard to aviation operations. Nonetheless, other mammals of similar (e.g., Mule deer) or smaller size (Coyote) have caused aircraft accidents in the U.S. White-tailed deer and Coyote are considered the first and $12^{\text {th }}$ most hazardous wildlife species to flight operations in the U.S. (FAA, 2018). Previous studies (DeVaul et al., 2018; Dolbeer, 2020) have identified an association between the wildlife body mass and the probability of a damaging strike. We could observe the presence of Cattle at Coe Field, their behavior, local movements, and preferred habitats. Additionally, our team could observe and record how cattle influence the presence and behavior of flocking birds. Both the visual and thermal cameras hold the potential to identify the presence of "large" mammals. Nonetheless, the "best" images were collected with the visual camera (see Figure 2).
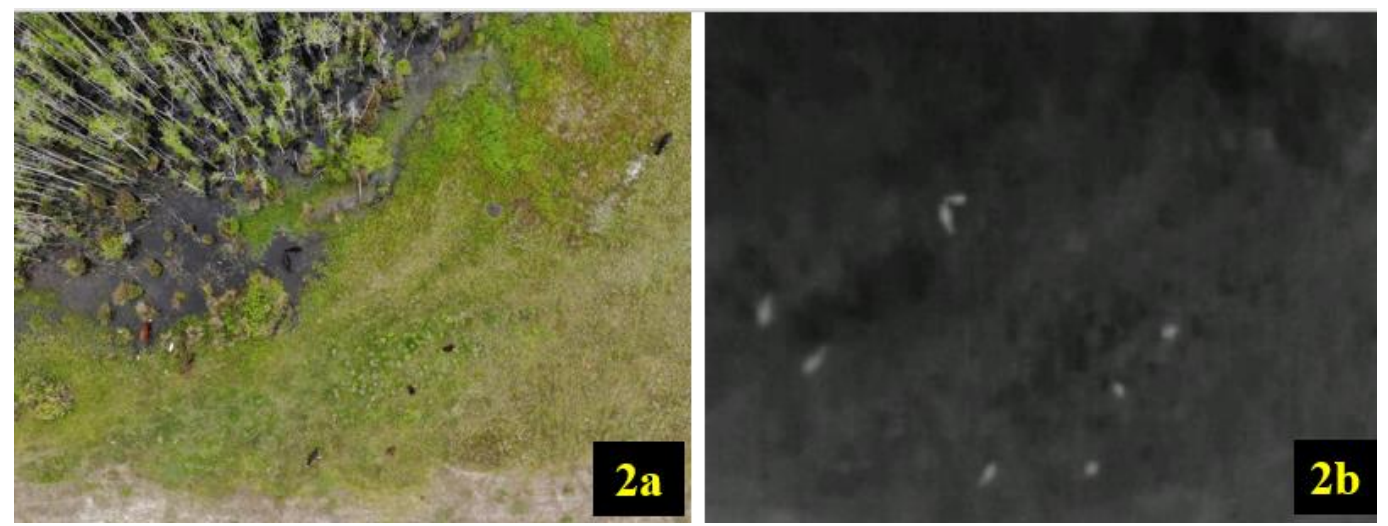

Figure 2. Images of Cattle at the Data Collection Area

Note. Figure $2 \mathrm{a}$ and $2 \mathrm{~b}$ were taken during a manual flight with the visual and thermal cameras, respectively.

There were 654 aviation safety occurrences involving Cattle egrets from 1990 through 2020 in the U.S. Forty-three and 66 of the total strikes caused damage to the aircraft and had a negative effect on flight (e.g., aborted takeoff), respectively. Almost 20\% $(n=124)$ of these strikes involved two or more animals. The FAA (2018) listed this bird species as the $14^{\text {th }}$ most hazardous wildlife species to aviation in the U.S. Cattle egrets forage in flocks, usually in fields and pastures where they can stalk small animals (e.g., insects) stirred from the ground (Cornell Lab of Ornithology, 2021c). Our team could observe, with the visual camera and especially during the manual flights, the presence, numbers, and behaviors of Cattle egrets. They were more abundant in the morning feeding on the ground and generally close to the cattle. We also observed them at or close to bodies of water. We could rarely "see" them with the thermal camera. It is important to mention that the drone allowed our team to observe these animals to include their numbers, behaviors, and local movements up to 500 meters from where the trailer was located. In different words, it would be difficult for a QAWB to thoroughly collect this information during a WHA even with binoculars due to the presence of shrubs and other elements between the trailer and the animals. Yet, the grass height can make it difficult for the QAWB to collect data and information on "small" birds that are foraging on the ground (see Figure 3). 

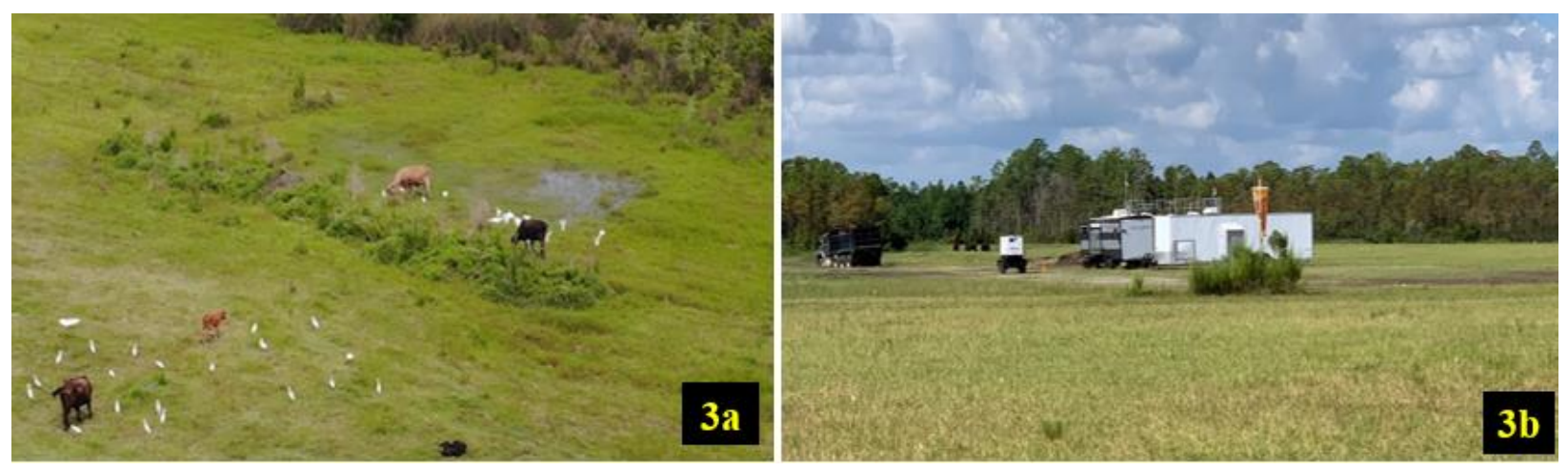

Figure 3. Images of Cattle Egrets the Data Collection Area

Note. Figure 3a shows 34 Cattle Egrets foraging close to cattle. Figure $3 \mathrm{~b}$ show man-made and other structures between the trailer and the location where we observed Cattle Egrets with the drone.

From 1990 through 2020 there were 178 strikes involving Sandhill cranes in the U.S. (Dolbeer et al., 2021). This bird species is listed as the fifth most hazardous wildlife species to aviation (FAA, 2018). This heavy-bodied animal's preferred habitats include pasture and prairies, and open grassland and wetlands. We could observe Sandhill cranes at Coe Field a few times with the UAS during the manual flights, usually during sunny weather. They were frequently in groups of three to five animals roaming in bodies of water or foraging close to wetlands.

"Land-use practices and habitat are the key factors determining the wildlife species and the size of wildlife populations that are attracted to airport environments" (FAA, 2018, p. 2-8). The identification of these land-use practices and habitats as well as the recording of the impact they have on the identified wildlife species is fundamental during a WHA. We could, using the visual camera during the autonomous and visual flights identify those land use-practices (e.g., livestock production) and habitats (e.g., wetland) and the relationship between the identified species and those sources of attraction. For example, Cattle in the data collection area is a factor determining the number and behavior of Cattle egrets (Cornell Lab of Ornithology, 2021). Moreover, our team observed and recorded the presence, numbers, and behaviors of Cattle egrets, Mottled ducks, Sandhill cranes, and White ibises being attracted to wetlands at Coe Field (Cornell Lab of Ornithology, 2021a, 2021b, 2021c). 


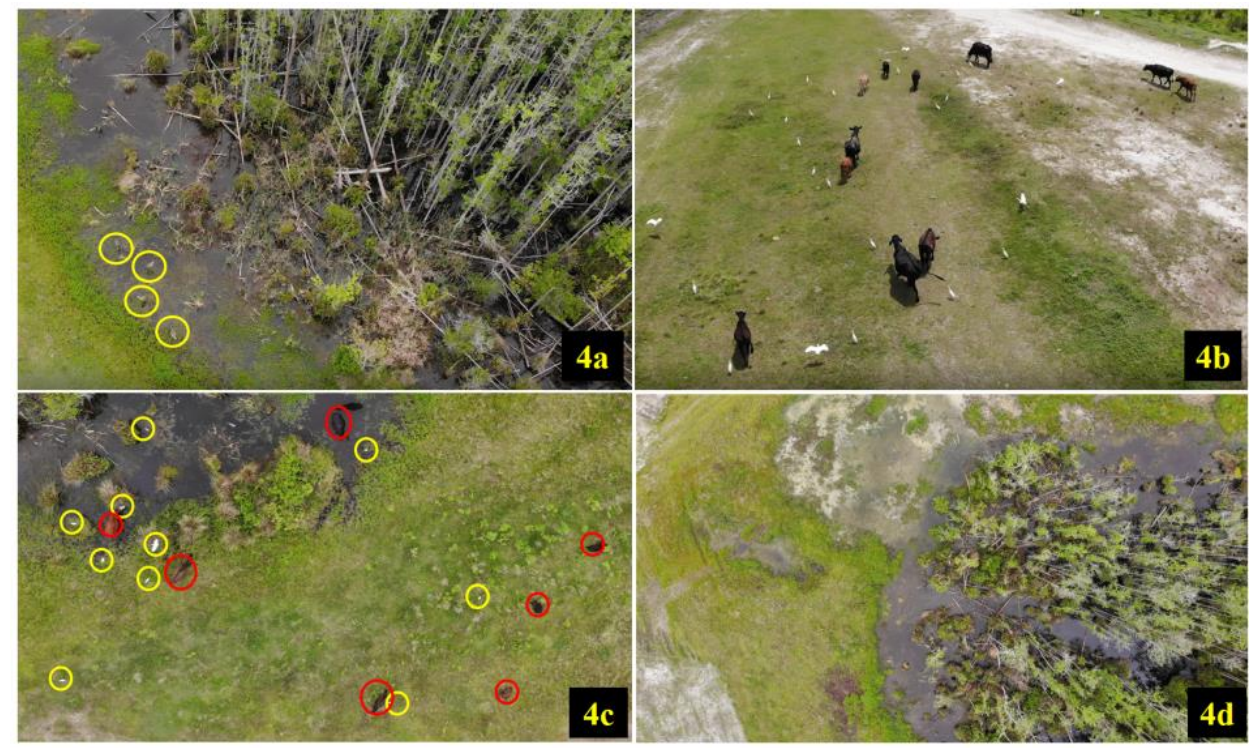

Figure 4. Multiple Images Collected with the UAS with the Visual Camera

Note. Figure 4a shows four Sandhill Cranes roaming in a Wetland. Figure $4 \mathrm{~b}$ shows the interactions between Cattle and Cattle Egrets at Coe Field. In Figure 4c, yellow and red circles indicate Cattle Egrets and Cattle, respectively that were attracted to a wetland. Figure 4d shows habitats with the potential to attract hazardous wildlife species to the airport environment.

\section{Conclusions}

The increased risk of mishaps due to wildlife strikes (Dolbeer et al., 2021) and the forecast growth for the U.S. aviation industry (FAA, 2020) require new strategies and great effort in order to continuously improve aviation safety. Multifaceted and innovative approaches to mitigate the risk of aircraft accidents resulting from strikes are fundamental. Findings from this ongoing study suggest that that UAS facilitates data collection in areas that are difficult to access by ground-based means (e.g., wetlands). Another key finding of this study was that our team could observe, and with the assistance of the QAWB identify different wildlife species and habitats simultaneously during each UAS flight. In different words, from a single image (video and/or picture) a QAWB could obtain valuable information about different wildlife species and related habitats. Moreover, results suggest that the versatility and speed of UAS (including their high-quality cameras and sensors) ensure that data can be collected more thoroughly and faster over large areas during a WHA. The use of UAS during a WHA can allow a QAWB to collect data that are difficult to access by ground-based means. Our findings also suggest that the risk associated with UAS operations at the airport environment can be reduced through the development of a ConOps that incorporates robust risk management processes and procedures. Findings also indicate that the ConOps must be periodically revised to incorporate procedures that improve aviation safety and efficiency. Most importantly, results suggest that the use of UAS during a WHA can enhance the data collection process by:

1. Collecting data and information on wildlife species, habitats, and land-uses at the airport environment faster and thoroughly; 

species;

2. Establishing a relationship between habitats and/or land uses and hazardous wildlife

3. Obtaining information of different wildlife species and habitats / land-uses simultaneously;

4. Obtaining information on wildlife species in areas that are difficult to access by ground-based means. 


\section{References}

Cabrera, J., Chimino, A., Woolf, N., Schwarz, M., \& Mendonca, F. A. C. (2021). Applying UAS for wildlife hazard management at airports. FAA challenge: Smart airport student competition. http://faachallenge.nianet.org/wpcontent/uploads/FAA_2021_TechnicalPaper_EmbryRiddleAeronauticalUniversity.pdf

Cleary, E. C., \& Dolbeer, R. A. (2005). Wildlife hazard management at airports: A manual for airport personnel. Federal Aviation Administration. http://www.faa.gov/airports/airport_safety/wildlife/resources/media/2005_faa_manual_co mplete.pdf

Cornell Lab of Ornithology (2021a). All about birds: Cattle Egret identification. The Cornell Lab. https://www.allaboutbirds.org/guide/Cattle_Egret/id

Cornell Lab of Ornithology (2021b). All about birds: Sandhill Crane identification. The Cornell Lab. https://www.allaboutbirds.org/guide/Sandhill_Crane/id

Cornell Lab of Ornithology (2021c). All about birds: Mottled Duck identification. The Cornell Lab. https://www.allaboutbirds.org/guide/Mottled_Duck/id

DeVault, on, H. A., Block, M. E., Wright, P. M., Ernst, D. M., Hayden, S. J., Lazzara, D. J., Savoy, S. M., \& Polston, E. K. (2007). Estimating interspecific economic risk of bird strikes with aircraft. Wildlife Society Bulletin 42(1), 94-101. https://digitalcommons.unl.edu/icwdm_usdanwrc/2074/

Dolbeer, R. A. (2020). Population increases of large birds in North America pose challenges for aviation safety. Human-Wildlife Interactions, 14(3), 345-357. https://doi.org/10.26077/53f9-edc3

Eschenfelder, P., \& DeFusco, R. (2010, August). Bird strike mitigation beyond the airport. AeroSafety World, 5(7). https://flightsafety.org/asw-article/bird-strike-mitigation-beyondthe-airport/

Federal Aviation Administration (FAA). (2016). Pilot's handbook of aeronautical knowledge. FAA. https://www.faa.gov/regulations_policies/handbooks_manuals/aviation/phak/

Federal Aviation Administration (FAA). (2018). Protocol for the conduct and review of wildlife hazard site visits, wildlife hazard assessments, and wildlife hazard management plans (AC 150/5200-38). FAA. https://www.faa.gov/documentLibrary/media/Advisory_Circular/150-5200-38.pdf 
Federal Aviation Administration (FAA). (2020). Hazardous wildlife attractants on or near airports (AC 150/5200-33C). FAA.

https://www.faa.gov/documentLibrary/media/Advisory_Circular/150-5200-33C.pdf

ForeFlight (2021). Internet traffic. ForeFlight: A Boeing company. https://foreflight.com/products/foreflight-mobile/internet-traffic/

Hamilton, B. A. (2020a). Airports and unmanned aircraft systems Volume 1: Managing and engaging stakeholders on UAS in the vicinity of airports (ACRP Research Report No. 212, volume 1). Transportation Research Board on the National Academies. https://www.nap.edu/catalog/25607/airports-and-unmanned-aircraft-systems-volume-3potential-use-of-uas-by-airport-operators

Hamilton, B. A. (2020b). Airports and unmanned aircraft systems Volume 3: Potential use of UAS by airport operators (ACRP Research Report No. 212, volume 3). Transportation Research Board on the National Academies. https://www.nap.edu/catalog/25607/airportsand-unmanned-aircraft-systems-volume-3-potential-use-of-uas-by-airport-operators

Mendonca, F. A. C., Carney, T. Q., \& Fanjoy, R. O. (2018). Enhancing the safety training of GA pilots to reduce the risk of bird strikes: An experimental pilot study. International Journal of Aviation, Aeronautics, and Aerospace, 5(4), 1-27. https://doi.org/10.15394/ijaaa.2018.1281

Neubauer, K., Fleet, D., Grosoli, F., \& Verstynen, H. (2015). Unmanned aircraft systems (UAS) at airports: A primer (ACRP Report 144). Transportation Research Board on the National Academies. https://www.nap.edu/catalog/21907/unmanned-aircraft-systemsuas-at-airports-a-primer

Prather, C. D. (2019). Current landscape of unmanned aircraft systems at airports (ACRP Synthesis 104). Transportation Research Board on the National Academies. http://www.trb.org/Main/Blurbs/180032.aspx 